\title{
Dissecting the function of networks underpinning language repetition
}

\author{
Marcelo L. Berthier ${ }^{*}$ and Matthew A. Lambon Ralph ${ }^{2}$ \\ ' Cognitive Neurology and Aphasia Unit, Centro de Investigaciones Médico-Sanitarias, University of Málaga, Málaga, Spain \\ ${ }^{2}$ Neuroscience and Aphasia Research Unit, School of Psychological Sciences, University of Manchester, UK \\ *Correspondence: mbt@uma.es
}

Edited and reviewed by:

John J. Foxe, Albert Einstein College of Medicine, USA

Keywords: repetition, arcuate fasciculus, dorsal stream, ventral stream, language, aphasia, conduction aphasia, temporal lobe

In the nineteenth century, ground-breaking observations on aphasia by Broca (1865) and Wernicke (1906) suggested that language function depends on the activity of the cerebral cortex. At the same time, Wernicke (1906) and Lichtheim (1885) also elaborated the first large-scale network model of language which incorporated long-range and short-range (transcortical connections) white matter pathways in language processing. The arcuate fasciculus (dorsal stream) was traditionally viewed as the major language pathway for repetition, but scientists also envisioned that white matter tracts traveling through the insular cortex (ventral stream) and transcortical connections may take part in language processing. Modern cognitive neuroscience has provided tools, including neuroimaging, which allow the in vivo examination of short- and long-distance white matter pathways binding cortical areas essential for verbal repetition. However, this state of the art on the neural correlates of language repetition has revealed contradictory findings, with some researchers defending the role of the dorsal and ventral streams, whereas others argue that only cortical hubs (Sylvian parieto-temporal cortex [Spt]) are crucially relevant.

An integrative approach would conceive that the interaction between these structures is essential for verbal repetition. For instance, different sectors of the cerebral cortex (e.g., Spt, inferior frontal gyrus/anterior insula) act as hubs dedicated to short-term storage of verbal information or articulatory planning and these areas in turn interact through forward and backward white matter projections. Importantly, white matter pathways should not be considered mere cable-like connections as changes in their microstructural properties correlate with focal cortical activity during language processing tasks.

Despite considerable progress, many outstanding questions await response. The articles in this Research Topic tackle many different and critical new questions, including: (1) how white matter pathways instantiate dialogues between different cortical language areas; (2) what are the specific roles of different white matter pathways in language functions in normal and pathological conditions; (3) what are the language consequences of discrete damage to branches of the dorsal and ventral streams; (4) what are the consequences (e.g., release from inhibition) of damage to the left white matter pathways in contralateral ones and viceversa; (5) how these pathways are reorganized after brain injury; (5) can the involvement/sparing of white matter pathways be used in outcome prediction and treatment response; and (5) can the microstructure of white matter pathways be remodeled with intensive rehabilitation training or biological approaches.

This Research Topic includes original studies, and opinion and review articles which describe new data as well as provocative and insightful interpretations of the recent literature on the role of white matter pathways in verbal repetition in normal and pathological conditions. A brief highlight summary of each is provided below.

\section{ARTICLES}

Opinion Article, Published on 29 Jul 2013

The anatomo-functional connectivity of word repetition: insights provided by awake brain tumor surgery

Sylvie Moritz-Gasser and Hugues Duffau

In this opinion article, Moritz-Gasser and Duffau (2013) provide important insights on the role of the strong interaction of dorsal and ventral streams in word repetition. The authors discuss their pioneering results obtained through direct electrical stimulation of white matter pathways in awake patients during brain tumor surgery.

Review Article, Published on 12 Jul 2013

Language repetition and short-term memory: an integrative framework

Steve Majerus

Short-term maintenance of verbal information is crucially important for efficient language repetition of complex information. In a comprehensive review, Majerus (2013) presents an integrative framework aimed at bridging research in the language processing and short-term memory fields.

Hypothesis and Theory Article, Published on 02 Oct 2013 Mapping a lateralization gradient within the ventral stream for auditory speech perception

Karsten Specht

Specht (2013) analyses the results from several complementary functional neuroimaging studies with the aim to trace the hierarchical processing network for speech comprehension within the left and right hemisphere. The author pays particular attention 
to role of the temporal lobe and the ventral stream in auditory speech perception.

Original Research Article, Published on 05 Sep 2013

Articulation-based sound perception in verbal repetition: a functional NIRS study

Sejin Yoo and Kyoung-Min Lee

Using functional near-infrared spectroscopy, Yoo and Lee (2013) examine healthy subjects while repeating pseudowords and words. This study reveals that passive listening without repetition to various sounds (natural environmental sounds, animal vocalizations, and human non-speech sounds) as well as articulation activate neural circuits that include both inferior frontal regions.

Original Research Article, Published on 26 Aug 2013

The roles of the "ventral" semantic and "dorsal" pathways in conduite d'approche: a neuroanatomically-constrained computational modeling investigation

Taiji Ueno and Matthew A. Lambon Ralph

In a computational modeling investigation of the dual dorsalventral pathway implicated in verbal repetition, Ueno and Lambon Ralph (2013) demonstrate that the successful phonetic approximations to target words (conduite d'approche), typically observed in patients with conduction aphasia and damage to the dorsal pathway (arcuate fasciculus), relies on the complementary activity of the ventral semantic stream.

Original Research Article, Published on 18 Oct 2013

Repeating with the right hemisphere: reduced interactions between phonological and lexical-semantic systems in crossed aphasia?

Irene De-Torres, Guadalupe Dávila, Marcelo L. Berthier, Seán Froudist Walsh, Ignacio Moreno-Torres and Rafael Ruiz-Cruces

In this study, De-Torres et al. (2013) show that repetition after subcortical lesions involving the dorsal and ventral streams in patients who are right-hemisphere dominant for language is not heavily influenced by lexical-semantic variables as is regularly reported in similar cases with left hemisphere damage.

Original Research Article, Published on 10 Dec 2013

Predicting speech fluency and naming abilities in aphasic patients

Jasmine Wang, Sarah Marchina, Andrea C. Norton, Catherine Y. Wan and Gottfried Schlaug

The identification of reliable biomarkers that predict the degree of chronic speech fluency/language impairment and potential for improvement after stroke is paramount. In this study, Wang et al. (2013) demonstrate that lesion load in the arcuate fasciculus (dorsal stream) is the best anatomical marker at stratifying patients into different outcome groups with high accuracy for speech fluency and naming.
Original Research Article, Published on 31 Jan 2014

Sensory-to-motor integration during auditory repetition: a combined fMRI and lesion study

'Ōiwi Parker Jones, Susan Prejawa, Tom Hope, Marion Oberhuber, Mohamed L. Seghier, Alex P. Leff, David W. Green and Cathy J. Price

On examining sensory-to-motor integration during auditory repetition in healthy subjects and aphasic patients, Parker Jones et al. (2014) find that normal and abnormal repetition of pseudowords correlate with activity of the arcuate fasciculus, but is unrelated to the activity of different cortical areas.

Original Research Article, Published on 19 Dec 2013

Dissociated repetition deficits in aphasia can reflect flexible interactions between left dorsal and ventral streams and gender-dimorphic architecture of the right dorsal stream

Marcelo L. Berthier, Seán Froudist Walsh, Guadalupe Dávila, Alejandro Nabrozidis, Rocio Juarez y Ruiz de Mier, Antonio Gutiérrez, Irene De Torres, Francisco Alfaro, Natalia GarcíaCasares and Rafael Ruiz-Cruces

Using multimodal neuroimaging, Berthier et al. (2013) evaluate the neural correlates of repetition performance in two aphasic patients matched for lesion volume (a female patient with preserved repetition and a male patient with impaired repetition). Dissociated repetition deficits in these cases are probably reliant on flexible interactions between left dorsal stream and left ventral stream and on gender-dimorphic architecture of the right dorsal stream.

Original Research Article

Dissecting the functional anatomy of auditory word repetition Thomas Matthew Hadley Hope, Susan Prejawa, 'Ôiwi Parker Jones, Marion Oberhuber, Mohamed L. Seghier, David W. Green and Cathy J. Price

Hope et al. (2013) use a single, multi-factorial, within-subjects fMRI design to identify those regions, and to functionally distinguish the multiple linguistic and non-linguistic processing areas, that are all involved in repeating back heard words. They find that repetition activates components of regions not hitherto implicated in word repetition. Thus, these novel findings challenge some of the commonly held opinions on the functional anatomy of language.

\section{REFERENCES}

Berthier, M. L., Walsh, S. F., Dávila, G., Nabrozidis, A., de Mier, R. J. R., Gutiérrez, A., et al. (2013). Dissociated repetition deficits in aphasia can reflect flexible interactions between left dorsal and ventral streams and gender-dimorphic architecture of the right dorsal stream. Front. Hum. Neurosci. 7: 873. doi: 10.3389/fnhum.2013.00873

Broca, P. (1865). Sur la faculté du langage articulé. Bull. Soc. Anthrop. (Paris) 6, 337-393.

De-Torres, I., Dávila, G., Berthier, M. L., Walsh, S. F., Moreno-Torres, I., and RuizCruces, R. (2013). Repeating with the right hemisphere: reduced interactions between phonological and lexical-semantic systems in crossed aphasia? Front. Hum. Neurosci. 7:675. doi: 10.3389/fnhum.2013.00675 
Hope, T. M. H., Prejawa, S., Parker Jones, O., Oberhuber, M., Seghier, M. L., Green, D. W., et al. (2013). Dissecting the functional anatomy of auditory word repetition. Front. Hum. Neurosci. 8:246. doi: 10.3389/fnhum.2014.00246

Lichtheim, L. (1885). On aphasia. Brain 7, 433-484.

Majerus, S. (2013). Language repetition and short-term memory: an integrative framework. Front. Hum. Neurosci. 7:357. doi: 10.3389/fnhum.2013.00357

Moritz-Gasser, S., and Duffau, H. (2013). The anatomo-functional connectivity of word repetition: insights provided by awake brain tumor surgery. Front. Hum. Neurosci. 7:405. doi: 10.3389/fnhum.2013.00405

Parker Jones, O., Prejawa, S., Hope, T., Oberhuber, M., Seghier, M. L., Leff, A. P., et al. (2014). Sensory-to-motor integration during auditory repetition: a combined fMRI and lesion study. Front. Hum. Neurosci. 8:24. doi: 10.3389/fnhum. 2014.00024

Specht, K. (2013). Mapping a lateralisation gradient within the ventral stream for auditory speech perception. Front. Hum. Neurosci. 7:629. doi: 10.3389/fnhum. 2013.00629

Ueno, T. and Lambon Ralph, M. A. (2013). The roles of the "ventral" semantic and "dorsal" pathways in conduite d'approche: a neuroanatomically-constrained computational modeling investigation. Front. Hum. Neurosci. 7:422. doi: 10.3389/fnhum.2013.00422

Wang, J., Marchina, S., Norton, A. C., Wan, C. Y., and Schlaug, G. (2013). Predicting speech fluency and naming abilities in aphasic patients. Front. Hum. Neurosci. 7:831. doi: 10.3389/fnhum.2013.00831
Wernicke, C. (1906). "Der aphasische Symptomencomplex," in Die duetsche Klinik am Eingange des 20: Jahrhunderts, Vol. 6, eds E. von Leyden and F. Klemperer (Berlin: Urban and Schwarzenberg), 487-566.

Yoo, S., and Lee, K.-M. (2013). Articulation-based sound perception in verbal repetition: a functional NIRS study. Front. Hum. Neurosci. 7:540. doi: 10.3389/ fnhum.2013.00540

Conflict of Interest Statement: The authors declare that the research was conducted in the absence of any commercial or financial relationships that could be construed as a potential conflict of interest.

Received: 28 August 2014; accepted: 29 August 2014; published online: 02 October 2014.

Citation: Berthier ML and Lambon Ralph MA (2014) Dissecting the function of networks underpinning language repetition. Front. Hum. Neurosci. 8:727. doi: 10.3389/ fnhum.2014.00727

This article was submitted to the journal Frontiers in Human Neuroscience.

Copyright (c) 2014 Berthier and Lambon Ralph. This is an open-access article distributed under the terms of the Creative Commons Attribution License (CC BY). The use, distribution or reproduction in other forums is permitted, provided the original author(s) or licensor are credited and that the original publication in this journal is cited, in accordance with accepted academic practice. No use, distribution or reproduction is permitted which does not comply with these terms. 